\title{
A systematic approach to multiphysics extensions of finite-element based micromagnetic simulations: Nmag
}

\author{
Thomas Fischbacher, Matteo Franchin, Giuliano Bordignon, Hans Fangohr \\ School of Engineering Sciences \\ University of Southampton, SO17 1BJ Southampton, United Kingdom \\ Email: fangohr@soton.ac.uk
}

\begin{abstract}
Extensions of the basic micromagnetic model that include effects such as spin-current interaction, diffusion of thermal energy or anisotropic magnetoresistance are often studied by performing simulations that use case-specific ad-hoc extensions of widely used software packages such as OOMMF or Magpar.

We present the novel software framework 'Nmag' that handles specifications of micromagnetic systems at a sufficiently abstract level to enable users with little programming experience to automatically translate a description of a large class of dynamical multifield equations plus a description of the system's geometry into a working simulation.

Conceptually, this is a step towards a higher-level abstract notation for classical multifield multiphysics simulations, similar to the change from assembly language to a higher level humanand-machine-readable formula notation for mathematical terms (FORTRAN) half a century ago.

We demonstrate the capability of this approach through two examples, showing (i) a reduced dimensionality model and use of arbitrary order shape functions, and (ii) the computation of a spatial current density distribution for anisotropic magnetoresistance (AMR).

For cross-wise validation purposes, we also show how Nmag compares to the $O O M M F$ and Magpar packages on a selected micromagnetic toy system. We furthermore briefly discuss the limitations of our framework and related conceptual questions.
\end{abstract}

\section{INTRODUCTION}

While there are various commercial and free software packages available for micromagnetical modeling and simulation, including the highly successful and popular open source packages $O O M M F$ [1] and Magpar [2], practically all the available packages run into limitations when one tries to couple magnetization dynamics with other classical field theory physics, as these software suites were designed as highly specialized frameworks for micromagnetism only. This means that in order to implement a particular multiphysics extension (such as the interaction of magnetization with electrical current), efficiency demands require either writing a considerable amount of low-level code to introduce additional fields, or alternatively writing (usually highly problem-dependent) glue code that translates simulation data back and forth between different packages. As nowadays there is growing demand to carry out multi-field physical simulations, we have embarked on the design and implementation of a new simulation system that treats micromagnetism as only one application of a generalized framework.

\section{DESIGN CONCEPTS}

Simulations in the abstract Nsim framework are set up by specifying physical and auxiliary fields that model scalar, vector, or (arbitrary-rank) tensorial quantities (like temperature, current, magnetization, angular momentum flow, etc.) as well as differential operators that provide relations between these fields, and furthermore local operators, in particular to implement (potentially non-linear) equations of motion.

As micromagnetism gave the incentive for the development of Nsim, most examples for the application of this package come from this field, and hence the micromagnetics part of Nsim - the Nmag [3] library - is the most evolved one in terms of more specialized speed-optimized functionality. Nevertheless, it is just as well possible to use Nsim to study other multiphysical models that do not include magnetization dynamics, e.g. coupling heat conduction with temperature dependent conductivity in a resistor. Likewise, Nsim can be used to couple the micromagnetic model to itself, modeling e.g. multicomponent magnetizations associated with different atomic species in an alloy.

The main objectives of Nsim are:

- Ease of use for casual (non-expert) users,

- Allowing self-contained problem descriptions, i.e. every simulation model should be completely specifiable in a single document that is both human- and computerreadable and as concise as possible.

- Providing a 'full generality' abstract multiphysics framework,

- Parallelizability.

This is achieved through:

- Providing a scripting language (Python [4]) interface which allows both batch processing as well as interactive use, and using a fast compiled language [5] internally.

- Abstract implementation of the underlying finite-element code that utilizes symbolic descriptions of elements and shape functions.

- Providing built-in (arbitrary dimension) mesh generation.

- An abstract mini-language for the specification of differential operators that also supports the Einstein summation convention. 
- Founding the package on MPI-based linear algebra and ODE solver libraries (PETSc [6] and Sundials [7]).

Whereas with packages like OOMMF and Magpar, the fundamental user-specifiable entities are the geometry and magnetical material parameters, Nsim in addition allows to introduce additional new fields governed by their own physical equations: basic micromagnetic models can be set up in Nsim by using the high-level Nmag library. We briefly review the structure of the general micromagnetic model to elucidate the abstract concepts supported by Nmag which are utilized to provide them.

The rate of change of the dynamical field is governed by a non-linear equation of motion with nontrivial index structure, the Landau-Lifschitz-Gilbert (LLG) equation:

$$
\frac{d \mathbf{M}}{d t}=-\frac{\gamma}{1+\alpha^{2}}\left(\mathbf{M} \times \mathbf{H}_{\mathrm{eff}}+\frac{\alpha}{|\mathbf{M}|} \mathbf{M} \times\left(\mathbf{M} \times \mathbf{H}_{\mathrm{eff}}\right)\right) .
$$

This equation gives rise to a conservation law $(d|\mathbf{M}| / d t=$ 0 ) which must be respected, but may be violated by numerical approximation errors. Basic support for user-definable equations of this general form is present in Nsim, but should be simplified and extended in future.

The auxiliary field $\mathbf{H}_{\text {eff }}$ that governs the dynamics receives contributions from various effects that are of very different nature:

$$
\mathbf{H}_{\text {eff }}=\mathbf{H}_{\text {ext }}+\mathbf{H}_{\text {anis }}+\mathbf{H}_{\text {exch }}+\mathbf{H}_{\text {demag }}+\text { other }
$$

Here, the external $\mathbf{H}_{\text {ext }}$ (Zeeman) magnetic field appears as an intensive thermodynamic variable: an 'external screw' that can be adjusted by the experimenter.

Concerning the specification of the anisotropy $\mathbf{H}_{\text {anis }}$, Nmag supports advanced techniques based internally on term manipulation which will be explained in detail in a future publication.

The exchange field contribution is a continuum approximation to the short-range neighbour-aligning Heisenberg spin-spin interaction and is computed by applying a multicomponent second order differential operator to $\mathbf{M}$ :

$$
H_{\mathrm{exch}, i}(\mathbf{x})=c \cdot \partial_{x_{j}} \partial_{x_{j}} M_{i}(\mathbf{x}) \quad\left(\text { i.e. } \mathbf{H}_{\mathrm{exch}}(\mathbf{x})=c \Delta \mathbf{M}(\mathbf{x})\right) \text {. }
$$

The Nmag library implements this operator via Nsim's support to map operator description strings to sparse matrices. ${ }^{1}$

The demagnetizing field $\mathbf{H}_{\text {demag }}$ introduces non-local couplings between all the magnetic moments in the simulation and hence is not computable via application of a sparsematrix-representable differential operator to the magnetization. Depending on the physical system, there are various techniques to compute $\mathbf{H}_{\text {demag }}$ (or approximations to it): for three-dimensional meshes with piecewise linear magnetization, Nsim can employ the hybrid finite element/boundary element method.

\footnotetext{
${ }^{1}$ The Nsim description of this particular operator is $"-c<d / d x j$ H_exch(i) ||d/dxj M(i) $>$, $i: 3, j: 3 "$
}

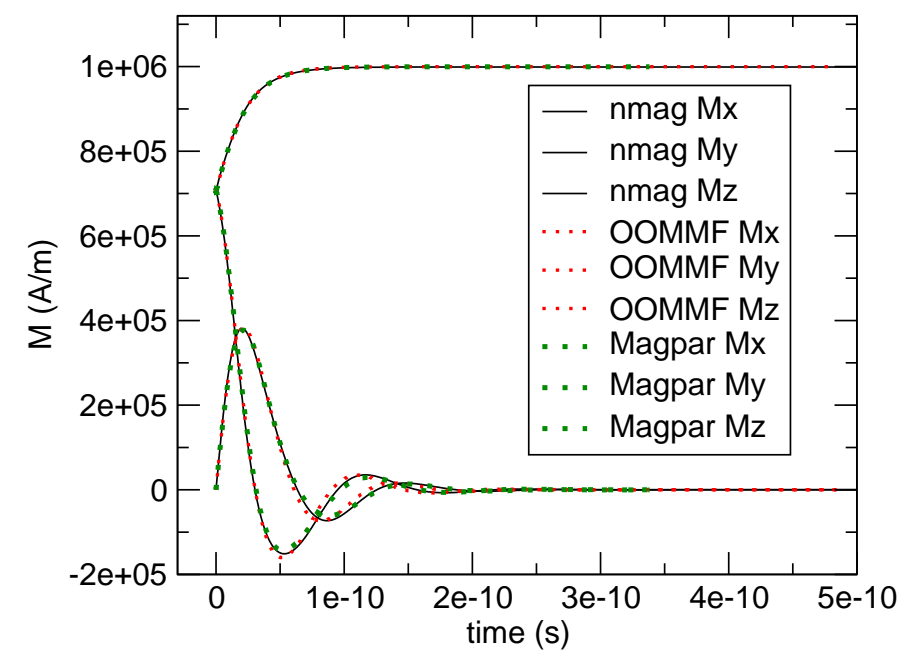

Fig. 1. Comparison of simulation packages for the bar example. Initial magnetization is $\left(M_{x}, M_{y}, M_{z}\right)=(\sqrt{2} / 2,0, \sqrt{2} / 2) \cdot 10^{6} \mathrm{~A} / \mathrm{m} . M_{z}$ increases over time, as the system tries to avoid strong magnetic effective surface charges on the long sides of the bar.

\section{Evaluation}

We use a simple validation example to compare simulation results obtained with (i) OOMMF, (ii) Magpar and (iii) Nmag: A ferromagnetic bar with dimensions $L_{\mathrm{x}, \mathrm{y}, \mathrm{z}}=$ $(10,10,50) \mathrm{nm}$, saturation magnetization of $M_{\mathrm{s}}=10^{6} \mathrm{~A} / \mathrm{m}$ and exchange constant $A=1.3 \cdot 10^{-11} \mathrm{~J} / \mathrm{m}$ is initially magnetized along the $(1,0,1)$ direction (i.e. 45 degrees between the $x$ and the $z$ axes). Fig. 1 shows the time development of the $x, y$ and $z$ components of the magnetization obtained with the aforementioned packages. We use a damping constant of $\alpha=0.5$.

Results are quantitatively very similar. Slight deviations are expected, as the various systems use different methods to discretise continuum physics, and employ different time integration schemes. In this example, magnetization aligns with the $z$-direction due to shape anisotropy of the bar.

\section{USAGE EXAMPLES}

\section{A. One dimension, higher order elements}

Fig. 2(left) shows the equilibrium magnetization configuration for a toy model resembling the system studied in [11]. Our system consists of an $x$-directed stack of three films of equal thickness. Assuming translational symmetry in the film plane, the system was reduced to one dimension, with $\mathbf{H}_{\text {demag }}$ simplifying to an additional anisotropy contribution. The planes form a sequence $\mathrm{AB}-\mathrm{AC}-\mathrm{AB}$ of binary alloys, where the atomic species $\mathrm{A}$ and $\mathrm{C}$ carry magnetic moments while B does not. C is exchange coupled only locally and anti-ferromagnetically to $\mathrm{A}$ and also experiences a strong quadratic uniaxial anisotropy, while there is no corresponding anisotropy term for A. We assume the A magnetization to be pinned in the $(0,1,0)$ as well as $(0,-1,0)$ direction on the left and right outer surfaces, respectively. Also, we 

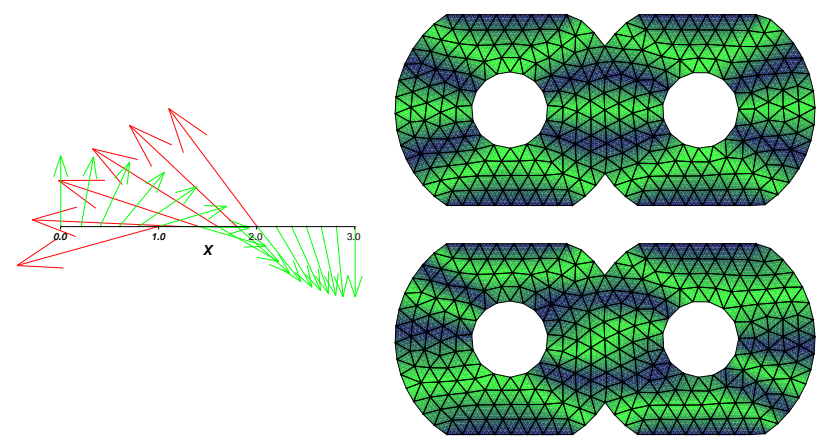

Fig. 2. Left: The film stack toy system's equilibrium magnetization for atomic species A and C. Right: Electrical potential of a toy AMR system, with greatly exaggerated coupling $\alpha=-0.9$ and constant magnetization $\mathbf{M}=(1,1)$. (Top: initial $\sigma(x)=\sigma_{0}$ configuration in the iterative solver, bottom: final equilibrium configuration). Computation done using 2 nd order elements. One color 'step' corresponds to $1 / 6$ of the total voltage across the sample.

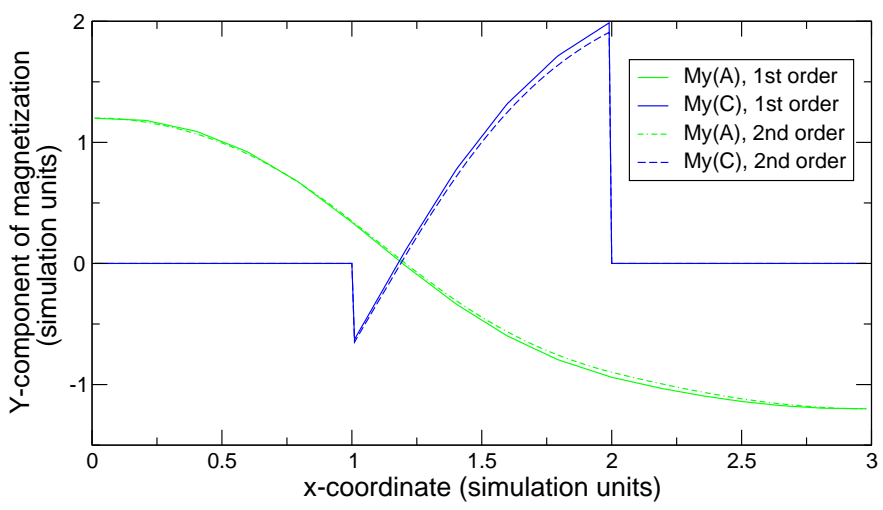

Fig. 3. Components $M_{y}$ of the film stack toy system's equilibrium magnetizations for atomic species $\mathrm{A}$ and $\mathrm{C}$, computed using 1st and 2nd order finite elements.

superimpose an external magnetic field in $+y$ direction. Parameters (in simulation units) for this example are (for interpretation, see [11]): $d_{\text {layer }}=1, M_{A}^{\text {sat. }}=1.2, M_{C}^{\text {sat. }}=2.5, J_{A A}^{\text {exch }}=4$, $J_{A C}^{\text {exch }}=-80, J_{C C}^{\text {exch }}=0, H_{\text {ext }}=(0,2.5,0), E_{C}^{\text {anis }}=4 M_{z}^{2}$.

Fig. 3 shows the equilibrium $M_{y}$ component for atomic species A and C, computed on a coarse 1-d 'mesh' of 33 sites, once using first-order elements, and once using second-order elements.

\section{B. Anisotropic Magnetoresistance}

Computation of the electrical current density $\mathbf{j}$ through a conductor with spatially dependent conductivity requires the solution of a generalized Laplace equation for the electrical potential $\Phi_{e}$, which is obtained from current conservation $\nabla \cdot \mathbf{j}=0$ and $\mathbf{j}=-\sigma \nabla \Phi_{e}$. The toy system presented in Fig. 2(right) is related to the system studied in [8] features both Dirichlet (fixed potential at top and bottom contacts) and von Neumann (no current leaving the sample through other boundaries) boundary conditions. Conductivity $\sigma(x)$ depends on the angle $\theta$ between current density $\mathbf{j}$ and magnetization $\mathbf{M}: \sigma(x)=\sigma_{0} /\left(1+\alpha \cos ^{2} \theta\right)$. The system is solved iteratively. Hall effect is not modeled in this toy example. Nsim supports a notion of operators containing spatial derivatives and products with other fields.

\section{LIMITATIONS OF OUR FRAMEWORK}

Nsim initially was designed to investigate the viability of the abstract approach towards modeling. Therefore, parts of the code that deal with initialization have not yet been optimised for speed. While the user may specify arbitrary equations of motion, the system does not yet recognise them at the abstract symbolic level, so auto-generation of code to compute the Jacobian for fast time integration ODE solvers is planned but so far unsupported. While Nsim is built on top of MPIaware libraries, core functionality is not yet parallelised: the main difficulty is that MPI imposes semantic restrictions on numerical code which require special tricks to support user interactivity at the same time. These limitations can be removed with reasonable implementation effort. Extending the system to support automatic adaptive mesh refinement at present would be difficult.

\section{CONCLUSION}

While large parts of the Nmag code are still very young and preliminary, first experience gained by using the system suggests that it can greatly increase productivity for setting up multiphysics simulations, in particular for systems with complicated physics but few degrees of freedom.

\section{ACKNOWLEDGMENT}

This works has been funded by the Engineering and Physical Sciences Research Council (EPSRC) in the United Kingdom (GR/T09156/01).

\section{REFERENCES}

[1] M. J. Donahue and D. G. Porter, OOMMF User's Guide, Version 1.0, National Institute of Standards and Technology, Gaithersburg, MD, Sept. 1999, interagency Report NISTIR 6376.

[2] W. Scholz, J. Fidler, T. Schrefl, D. Suess, R. Dittrich, H. Forster, and V. Tsiantos, "Scalable parallel micromagnetic solvers for magnetic nanostructures," Comp. Mat. Sci, vol. 28, pp. 366-383, 2003.

[3] Nmag - a micromagnetic simulation environment, http://www. soton.ac.uk/ fangohr/nsim/nmag.

[4] G. van Rossum, "Python website," 2006, http://www. python.org.

[5] X. Leroy, D. Doligez, J. Garrigue, D. Rémy, and J. Vouillon, "Objective caml website," 2006, http:// caml.inria.fr.

[6] S. Balay, W. D. Gropp, L. C. McInnes, and B. F. Smith, "Efficient management of parallelism in object oriented numerical software libraries," in Modern Software Tools in Scientific Computing, E. Arge, A. M. Bruaset, and H. P. Langtangen, Eds. Birkhauser Press, 1997, pp. 163-202.

[7] P. Brown, A. Collier, K. Grant, A. Hindmarsh, S. Lee, R. Serban, and C. Woodward, "Sundials (SUite of Nonlinear and DIfferential/ALgebraic equation Solvers home page, version 2.2," 2006, http://www.llnl.gov/CASC/sundials/.

[8] G. Bordignon, T. Fischbacher, M. Franchin, J. P. Zimmermann, A. Zhukov, P. de Groot, and H. Fangohr, "Analysis of magnetoresistance in arrays of connected nano-rings," IEEE Transactions on Magnetics, 2007, (in print) 
[9] D. R. Fredkin and T. R. Koehler, "Hybrid method for computing demagnetizing fields," IEEE Trans. Magn., vol. 26, pp. 415-417, 1990.

[10] D. A. Lindholm, "Three-dimensional magnetostatic fields from pointmatched integral equations with linearly varying scalar sources," IEEE Trans. Magn., vol. 20, pp. 2025-2032, 1984.

[11] M. Franchin, J. Zimmermann, T. Fischbacher, G. Bordignon, P. de Groot, and H. Fangohr, "Micromagnetic modelling of the dynamics of exchange springs in multi-layer systems," IEEE Transactions on Magnetics, 2007, (in print). 